\title{
El factor demográfico en la sostenibilidad del sistema de pensiones en España
}

\section{Albert Esteve, Daniel Devolder y Amand Blanes, Centre d'Estudis Demogràfics}

La caída de los nacimientos, el aumento de la esperanza de vida y la jubilación futura de los baby-boomers desafía la sostenibilidad del sistema de pensiones en España porque disminuye la relación entre cotizantes y jubilados. En este número de Perspectivas Demográficas examinamos la incidencia de estas causas sobre la sostenibilidad demográfica del sistema y el efecto que distintos escenarios de mortalidad, fecundidad y migración exterior podrían tener en el futuro. Las condiciones demográficas del pasado, muy favorables para el sistema, no se repetirán en el futuro, pero la demografía que viene no debería ser obstáculo para mantener un sistema de pensiones basado en la idea de reparto, sostenible, solidario y suficiente.

\section{HOY POR TI, MAÑANA POR MI}

El sistema de pensiones en España está basado en un pacto solidario entre generaciones. Las personas ocupadas generan cotizaciones que, gestionadas por la Seguridad Social, se utilizan para pagar las pensiones de las personas que en el pasado adquirieron el derecho a percibir una pensión vitalicia de jubilación. La sostenibilidad del sistema depende de la diferencia entre el volumen de las cotizaciones y el de las pensiones. Éstos a su vez dependen, principalmente, de factores económicos y demográficos. Entre los económicos destacan las tasas de ocupación y el nivel de salarios, estrechamente relacionados con la productividad de la economía. Y, entre los demográficos, los factores más importantes son el tamaño y la duración de las cohortes de nacimiento. El tamaño se refiere al número de personas que constituye una cohorte, que es resultado de la fecundidad/nacimientos del pasado, la mortalidad y las salidas y entradas por migración. La duración corresponde a la vida media que transcurre desde que nace una cohorte hasta que fallecen todos sus miembros y, a efectos del cálculo de las pensiones, desde que se jubilan hasta que mueren. Tamaño y duración condicionan la relación entre la población activa/ocupada y la jubilada. Cuanta más población ocupada hay respecto a la jubilada, más sostenible es el sistema. El persistente aumento de la esperanza de vida y la caída y estancamiento de la fecundidad por debajo de 1,5 hijos por mujer disminuye -y lo seguirá haciendo- la relación entre población en edad de trabajar y jubilados, coyunturalmente aliviada por la incorporación al mercado de trabajo de las generaciones llenas nacidas entre 1960 y 1975, los baby-boomers. No obstante, la jubilación cercana de los baby-boomers junto con la entrada de generaciones vacías al mercado laboral ha disparado las alarmas sobre la sostenibilidad del sistema de pensiones en España y en la mayoría de países occidentales.

\section{LA SOSTENIBILIDAD DEMOGRÁFICA DEL SISTEMA DE PENSIONES: PASADO Y PRE- SENTE}

El Indicador de Sostenibilidad Demográfica (ISD) del sistema de pensiones mide la relación entre el volumen de cotizaciones y el volumen de pensiones de jubilación en función de la demografía (distribución por edad y sexo) de cada momento (Fig. 1). Este indicador asume un perfil económico y constante en el tiempo.

FIGURA 1. La sostenibilidad demográfica del sistema de pensiones en España, 1970-2070

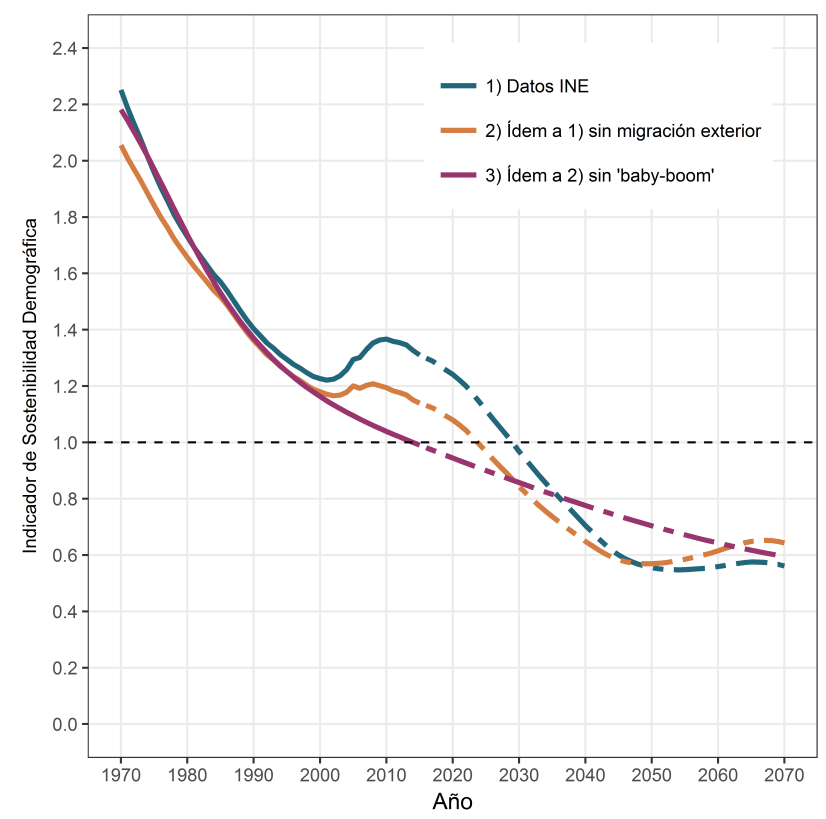

Fuente: Elaboración con datos INE y cálculos propios. 
Asumimos aquí la estructura de renta laboral, las tasas de ocupación y de desempleo, las cotizaciones y las pensiones de 2012 constantes en el tiempo, retrospectiva y prospectivamente. En ese año, la cotización media representaba el 23\% del salario y el importe de la jubilación equivalía en promedio al 60\% del último salario. El sistema es sostenible (ISD igual o mayor a 1) cuando las cotizaciones generadas por la población cotizante son iguales o superiores a las pensiones demandadas por la población receptora de pensiones. Obviamente, el nivel absoluto del indicador varía en función del perfil económico de referencia, pero para poder aislar el efecto demográfico es preciso utilizar un único perfil de referencia. Por tanto, nos centraremos más en la evolución del indicador en el tiempo que en sus valores absolutos.

La Figura 1 muestra la evolución del ISD entre 1970 y 2070 en función de tres escenarios. El primer escenario está basado en la evolución demográfica observada hasta 2015 y la proyectada se basa en el escenario medio de las proyecciones publicadas por el INE en 2016. El segundo escenario pregunta qué le hubiera ocurrido al indicador de sostenibilidad sin la inmigración internacional llegada a España en las últimas dos décadas. El tercero añade una suposición adicional: sustituye la evolución de los nacimientos observados por una evolución tendencial y moderada de los nacimientos en el pasado. Los tres escenarios muestran que España se ha beneficiado de unas condiciones demográficas muy favorables para el mantenimiento del sistema de pensiones. Asumiendo el perfil económico de 2012, las cotizaciones en 1970 hubieran sumado el doble de ingresos de lo que el sistema demandaba en pensiones de jubilación. Sin embargo, entre 1970 y 2000, el indicador de sostenibilidad decrece hasta el 1,2 (las cotizaciones son un 20\% superiores a las pensiones). La caída del indicador se detiene y el nivel se mantiene por encima de 1,2 hasta el año 2022 gracias a la incorporación de las generaciones del baby-boom al mercado de trabajo y a la llegada de la inmigración internacional. Sin babyboom y sin inmigración internacional las tensiones demográficas en el sistema de pensiones serían hoy mayores, en concreto el ISD sería cerca de un 30\% más bajo. Es importante observar también que, desde una perspectiva estrictamente demográfica, la sostenibilidad del sistema sería hoy mayor que la del año 2000.

\section{TENDENCIAS (¿Y SOLUCIONES DEMOGRÁ- FICAS?) A CORTO Y MEDIO PLAZO}

Los paneles de la Figura 2 representan el indicador de sostenibilidad demográfica proyectado hasta el año 2070 según diferentes escenarios de futuro sobre la evolución de la mortalidad / esperanza de vida (panel 1), la fecundidad (panel 2) y las migraciones internacionales (panel 3). Si la mortalidad se mantuviera constante en el futuro al nivel de 2015, la sostenibilidad demográfica del sistema de pensiones caería hasta 0,72 en 2045 (contribuciones inferiores en un $28 \%$ a las pensiones). Si aumentara la esperanza de vida en el futuro al ritmo de las últimas décadas, el indicador de sostenibilidad caería hasta o,6 en 2045. El 83\% del descenso de la sostenibilidad demográfica hasta el 2045 es directamente atribuible al efecto de la variación del tamaño de las cohortes que se jubilan (los babyboomers) y el 17\% al aumento de la esperanza de vida (su duración). Este cálculo resulta de comparar la disminución del ISD entre 2015 y 2045 sin y con aumento de la esperanza de vida.

Para medir el impacto de la recuperación de la fecundidad sobre la sostenibilidad demográfica del sistema de pensiones barajamos dos escenarios de crecimiento de la fecundidad (ver Figura 2 panel 2). En el primero, la fecundidad crece tendencialmente de 1,33 hijos

FIGURA 2. La sostenibilidad demográfica futura del sistema de pensiones según diferentes escenarios sobre la mortalidad, la fecundidad y la migración
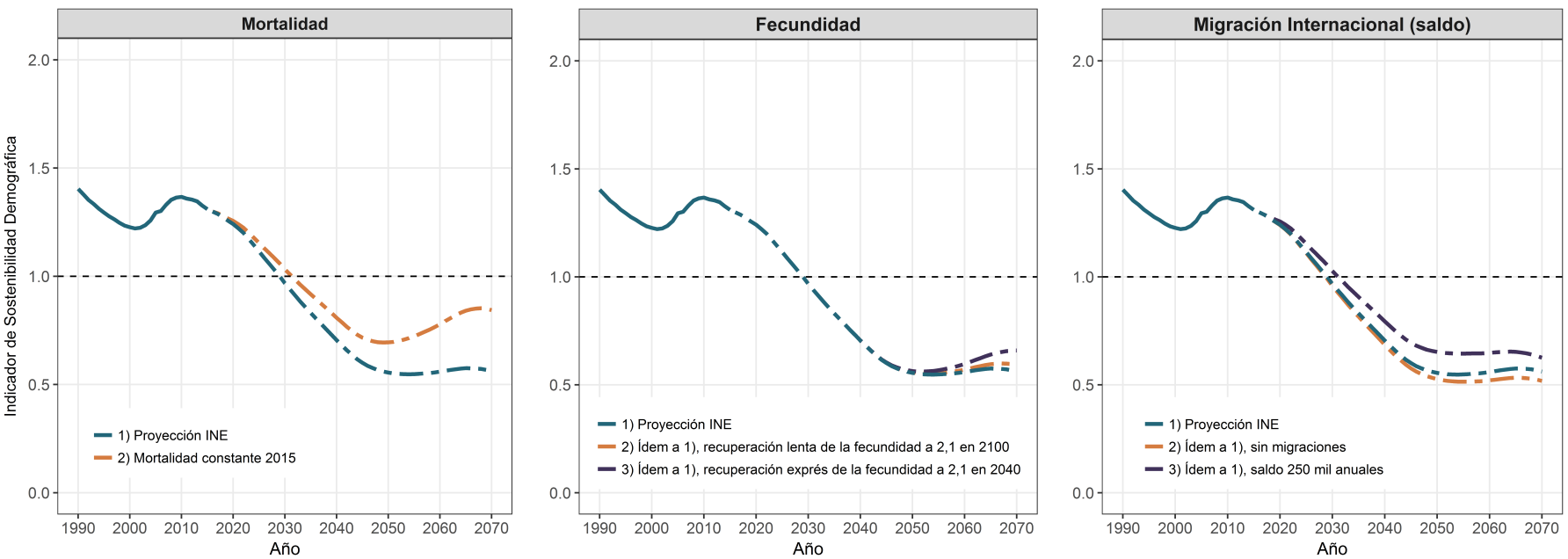

Fuente: Elaboración con datos INE y cálculos propios del INE. 
por mujer a 2,1 entre 2016 y 2100. En el segundo, la fecundidad alcanza los 2,1 hijos por mujer en 2040 y posteriormente se mantiene constante en ese nivel. La recuperación de la fecundidad tiene efectos a largo plazo sobre la sostenibilidad demográfica del sistema, pero son imperceptibles antes de 2050. La recuperación exprés de la fecundidad mejoraría la sostenibilidad del sistema en 2070 aunque no sería suficiente para revertir su caída.

La inmigración tiene a corto plazo efectos positivos sobre la sostenibilidad demográfica del sistema (ver panel 3 Figura 2). Sin embargo, a medio y a largo plazo los inmigrantes también se jubilan. Un saldo migratorio moderadamente positivo y creciente en el tiempo, de 12 mil personas/año en 2016 a 80 mil a partir de 2065 según la última previsión del INE mejoraría ligeramente los índices de sostenibilidad respecto al escenario sin crecimiento migratorio. Si el saldo aumentara hasta cifras de 250 mil personas anuales, el indicador crecería en 10 puntos hasta el año 2050 (de 0,55 hasta 0,65). A largo plazo, en 2070, ningún escenario es suficiente para revertir la caída del indicador de sostenibilidad.

\section{LA EDAD A LA JUBILACIÓN}

Desde un punto de vista teórico, retrasar la edad a la jubilación es una solución sencilla para garantizar la sostenibilidad demográfica del sistema de pensiones. Con ello, el periodo de cotización se alarga y el de jubilación se acorta. La Figura 3 muestra la edad a la jubilación que mantendría el sistema en equilibrio según dos escenarios. El primer escenario refleja las condiciones demográficas observadas entre 1970 y 2015 y las proyectadas por el INE hasta 2070. En 1970, gracias a las favorables condiciones demográficas del país, la edad de jubilación hubiera podido fijarse en los 56,8 años, 4,5 años menos que la estimada en 2016. La incorporación plena de los baby-boomers al mercado de trabajo, sumada a la llegada de la inmigración internacional, hubiera permitido disminuir la edad a la jubilación de 62,3 en 1999 a 60,7 en 2009, año a partir del cual crecería hasta los 73,8 en 2058 cuando las generaciones del baby-boom estarán completamente jubiladas. Las oscilaciones observadas en la edad a la jubilación de equilibrio reflejan la entrada y salida del mercado de trabajo de cohortes de distinto tamaño.

El segundo escenario refleja la evolución de la edad a la jubilación de equilibrio en un modelo de población que repite las condiciones de mortalidad del primer escenario, pero asume una tendencia de evolución de los nacimientos entre el -0,2\% y el 0,2\% anual. Este modelo de población no está sujeto a variaciones bruscas en el número de nacimientos debido, por ejemplo, a los efectos coyunturales del baby-boom. Tampoco contempla salidas y entradas por
FIGURA 3. Edad a la jubilación necesaria para mantener el sistema en equilibrio según diferentes escenarios de población

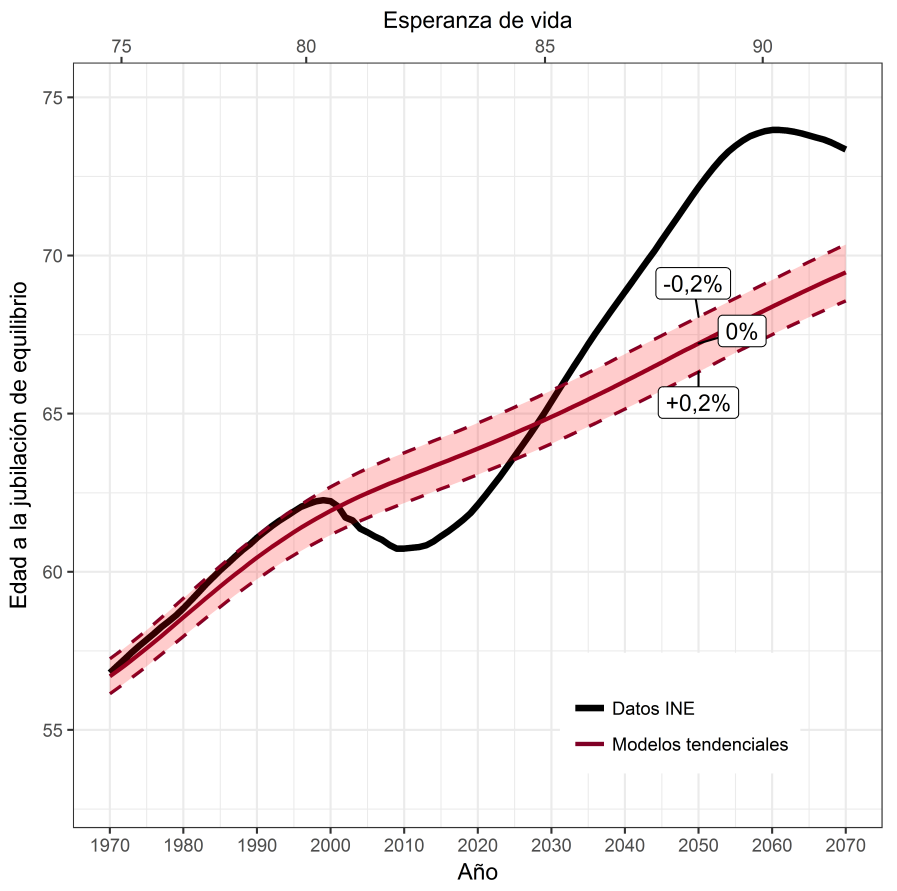

Fuente: Elaboración con datos INE y cálculos propios.

Nota: La curva negra está basada en la población observada hasta 2015 y la proyectada por el INE. La curva roja está basada en un modelo que tiene en cuenta principalmente el cambio en la mortalidad sin migraciones ni vaivenes en la natalidad.

migración. La diferencia en la edad de jubilación de equilibrio entre el umbral de mayor crecimiento $(0,2)$ y el de menor $(-0,2)$ es de 2 años. En este modelo, la edad a la jubilación de equilibrio aumenta según crece la esperanza de vida. En el año 2030, la edad de jubilación de equilibrio se situaría en los 65 años en los dos escenarios. A partir de este año, la jubilación de los baby-boomers obligaría a retrasar la edad a la jubilación a un ritmo mayor de lo que sería necesario si sólo tuviéramos en cuenta el aumento de la esperanza de vida. En el año 2050, la diferencia entre un escenario con o sin baby-boomers es de casi 6 años.

\section{LA VIABILIDAD DEMOGRÁFICA DEL SISTE- MA: UNA REFLEXIÓN DE CONJUNTO}

En los últimos cuarenta años, la sociedad española ha consolidado un sistema de pensiones basado en la idea de reparto sobre unas condiciones demográficas irrepetiblemente favorables. Unas condiciones basadas en el crecimiento continuado de la población activa, en una edad a la jubilación relativamente alta en relación a los niveles de esperanza de vida de la época, en la jubilación de unas cohortes escasas y castigadas por una mortalidad más elevada que la actual. Frente a la jubilación de los baby-boomers, el crecimiento de la esperanza de vida y la entrada al mercado laboral de genera- 
ciones vacías, el futuro depara unas condiciones radicalmente distintas. En consecuencia, la sostenibilidad venidera del sistema de pensiones no podrá apoyarse en la demografía. Ni una poco probable recuperación exprés de la fecundidad ni un saldo migratorio positivo de hasta 250 mil entradas anuales podrían revertir el efecto en el sistema de la jubilación de los baby-boomers, y del crecimiento de la esperanza de vida. Sin embargo, esto no significa que la demografía del futuro sea un obstáculo para la viabilidad del sistema de pensiones. Ajustar los periodos de cotización y jubilación en función de la esperanza de vida es una medida razonable para cuadrar las cifras. Ahora bien, este ajuste debería basarse exclusivamente en las ganancias de esperanza de vida y no en las necesidades del sistema para cuadrar las cuentas ante la jubilación de generaciones de mayor o menor tamaño. En efecto, penalizar unas generaciones sobre otras por su tamaño constituiría claramente una inequidad intergeneracional.

En este ejercicio hemos dejado intencionadamente al margen el impacto de las variaciones económicas en el sistema para aislar el efecto del cambio demográfico de forma nítida. Lógicamente, si modificáramos los parámetros económicos, los niveles de sostenibilidad demográfica del sistema de pensiones variarían. Por ejemplo, según nuestro modelo, el aumento de un punto porcentual en el nivel de cotización, es decir, pasar del 23\% al 24\% del salario, permitiría reducir entre 5 y 7 meses la edad de equilibrio a la jubilación y retrasaría entre 3 y 5 años el momento en el que el sistema entraría en déficit. La economía española tiene amplio recorrido para mejorar sus niveles de productividad y actividad. El aumento de la actividad y ocupación entre los jóvenes y la población mayor de 60 años o la igualación de las tasas de actividad femenina y masculina tendrían efectos positivos sobre la sostenibilidad del sistema a corto y a medio plazo. Si la economía española sabe capitalizar y mejorar la productividad de sus activos, la demografía no pondrá obstáculos a la viabilidad del sistema.

La demografía de las altas esperanzas de vida, de los nacimientos menguantes, del crecimiento de la población a expensas de los movimientos migratorios, $\mathrm{y}$ de las pirámides transformadas en obeliscos ha venido para quedarse. Pero esto no implica que los sistemas de pensiones basados en la idea de reparto no sean sostenibles en estas condiciones. Para ello, habrá que adecuar los periodos de trabajo y jubilación a los incrementos netos de esperanza de vida de una forma justa y equitativa con el tipo de trabajo y el esfuerzo que han realizado las generaciones en el pasado, con independencia de su tamaño. La sostenibilidad futura exige mejoras en la productividad de la economía y cambios en la provisión económica del sistema. De lo contrario, la confianza de la ciudadanía en el sistema de pensiones mermaría. Esto supondría un desafío para el sistema de mayor alcance que el demográfico.
Referencias bibliográficas

BLANES, Amand; CABRÉ, Anna (2015) "Inercias e incertidumbres en el futuro demográfico de España”. Revista del Ministerio de Empleo y Seguridad Social, 119 ("Seguridad Social”): 105-129.

FERNÁNDEZ CORDÓN, Juan Antonio (2015) "Relaciones intergeneracionales, demografía y economía en relación con las pensiones", Cuadernos de relaciones laborales, 33 (2) 235-258.

INSTITUTO NACIONAL DE ESTADÍSTICA (2016) "Proyecciones de la población de España, 2016-2066. Metodología", Madrid, www.ine.es.

LEE, Ronald D; MASON, Andrew (2011) "Population Aging and the Generational Economy: A Global Perspective", Cheltenham, Edward Elgar.

\section{Cita}

Albert Esteve, Daniel Devolder y Amand Blanes (2018) "El factor demográfico en la sostenibilidad del sistema de pensiones en España", Perspectives Demogràfiques, 9: 1-4.

Editores

Andreu Domingo y Albert Esteve

Correspondencia dirigida a

Albert Esteve

aesteve@ced.uab.es

Créditos

Gráficos: Anna Turu

Maquetación: Xavier Ruiz Vilchez

Agradecimientos

Elisenda Rentería y Rocío Treviño

\section{Enlace url}

http://ced.uab.es/es/difusion/ butlleti-perspectives-demografiques

\section{Contacto}

Centre d'Estudis Demogràfics.

Calle de Ca n’Altayó, Edificio E2

Universitat Autònoma de Barcelona

08193 Bellaterra / Barcelona

España

Teléfono: +34 935813060

E-mail:demog@ced.uab.es

Web: http://ced.uab.es/es/ 